Article

\title{
The Effect of Laser Pulse Widths on Laser-Ag Nanoparticle Interaction: Femto- to Nanosecond Lasers
}

\author{
Jin-Woo Jeon ${ }^{1}$, Sangwoo Yoon ${ }^{2}$, Hae Woon Choi ${ }^{3}$, Joohan Kim ${ }^{2, *}$, Dave Farson ${ }^{4}$ \\ and Sung-Hak Cho 1,5 \\ 1 Department of Laser \& Electron Beam Application, Korea Institute of Machinery \& Materials, Daejeon 34103, \\ S. Korea; jwj@kimm.re.kr (J.-W.J.); shcho@kimm.re.kr (S.-H.C.) \\ 2 Department of Mechanical Engineering, Seoul National University of Science and Technology, Seoul 01811, \\ S. Korea; yoonsw@seoultech.ac.kr \\ 3 Department of Mechanical and Automotive Engineering, Keimyung University, Daegu 42601, S. Korea; \\ hwchoi@kmu.ac.kr \\ 4 Department of Material Science Engineering, the Ohio State University, Columbus, OH 43210, USA; \\ farson.4@osu.edu \\ 5 Department of Nano-Mechatronics, Korea University of Science \& Technology, Daejeon 34113, S. Korea \\ * Correspondence: joohankim@seoultech.ac.kr; Tel.: +82-2-970-6305
}

Received: 14 December 2017; Accepted: 11 January 2018; Published: 14 January 2018

\begin{abstract}
The effect of the laser pulse width on the production of nanoparticles by laser fragmentation was investigated. Laser pulse widths of $164 \mathrm{fs}, 5 \mathrm{ps}, 4 \mathrm{~ns}, 36 \mathrm{~ns}, 64 \mathrm{~ns}$, and $100 \mathrm{~ns}$ were used. To assess the effect of the laser pulse width on the energy distribution in the nanoparticles, the energy distribution was simulated using wave optics. Silver (Ag) nanoparticles were produced by laser irradiation of an Ag target in distilled water. The wavelength of the femtosecond, picosecond, and nanosecond lasers used was $1070 \mathrm{~nm}$, and their fluences were $0.10-0.13 \mathrm{~mJ} / \mathrm{cm}^{2}$. Nanoparticle microstructure was visualized by transmission electron microscopy and scanning electron microscopy, and the nanoparticle size distribution was evaluated using a particle size analyzer.
\end{abstract}

Keywords: laser fragmentation; laser pulse width; nanoparticles

\section{Introduction}

Silver (Ag) nanoparticles are used for a number of applications because of their electrical and biochemical properties [1-3]. Therefore, research has focused on the optical, electrical, physical, and chemical properties of Ag nanoparticles [4-6]. For example, carbon nanotubes and Ag nanoparticles have been used as additives to improve the properties of materials for transparent electrodes or solar panels, for which light transmittance and electrical conductivity are important [7-11]. The effect on the properties of composites of the addition of nanostructures was also examined [12]. $\mathrm{Ag}$ in the form of nanowires has been used to improve the light transmittance and electrical conductivity of target materials $[13,14]$.

Chemical- and laser-based methods for the synthesis of $\mathrm{Ag}$ nanostructures have been developed [15-19]. Among them, laser fragmentation has advanced with developments in laser sources. Laser fragmentation proceeds by irradiating a bulk metal target in a liquid with a laser pulse. This causes the ablation of the target surface, and the debris generated are degraded into nanoparticles by laser irradiation $[20,21]$. Compared to conventional chemical synthesis methods, laser fragmentation reduces the risk of contamination of the particles during processing. In addition, this method generates nanoparticles with a purity greater than that of colloidal nanomaterials made by conventional chemical synthesis methods. Because laser fragmentation involves the direct laser irradiation of particles in 
a liquid, it has greater energy transfer efficiency than laser ablation of materials in air. The synthesis of alloy nanoparticles and the production of various 3D nanoparticles in a porous form using laser ablation and laser fragmentation in water were reported [22,23]. Also, heat is efficiently transferred from the particles to the liquid. The mechanical pressure waves generated in the liquid also affect particle generation $[24,25]$.

Laser fragmentation facilitates the control of process parameters and enables the production of nanoparticles of various qualities [26]. In particular, the size of the resulting nanoparticles can be controlled by varying the laser pulse width or fluence. For example, Maximova et al. [27] produced nanoparticles of different sizes using femtosecond laser pulses and described the mechanism of laser fragmentation. Another research group investigated the effect on particle distribution and size of varying laser wavelengths and presented the principle of laser fragmentation [28]. It was also reported that a transferring wire target in a liquid jet can produce nanoparticles of a certain size at a high synthesis rate [29].

Irradiation by a femtosecond laser pulse width generally induces nonlinear multiphoton absorption by the nanoparticles [30]. In contrast, particle fragmentation by thermal ablation occurs mainly by melting and evaporation during irradiation by laser pulse widths in the nanosecond range [31]. Studies on the effect of the laser pulse width on laser fragmentation have mostly been conducted independently, using femtosecond, picosecond, or nanosecond pulse widths [20,32-35]. In addition, various laser fragmentation processes and metal targets can be used to produce nanoparticles. Examples of laser process parameters and nanoparticle sizes are shown in Table 1. However, these studies have several drawbacks. For example, the laser energy density was in the femtosecond or picosecond range. Also, the use of different laser pulse energies or fluences hampers the comparison of the results of different studies. Link et al. [32] assessed the effects of laser pulse energy and pulse width on nanoparticle generation for Au nanorods. They transformed Au nanorods into Au nanoparticles using femtosecond and nanosecond laser pulse widths.

Table 1. Characteristics of the nanoparticles produced using various laser parameters.

\begin{tabular}{cccccc}
\hline Material & Wavelength & Pulse Width & Fluence or Pulse Energy & Particle Size & Reference \\
\hline $\mathrm{Au}$ & 400 and $532 \mathrm{~nm}$ & $150 \mathrm{fs}$ & 7.3 and $3.6 \mathrm{~mJ} / \mathrm{cm}^{2}$ & $\sim 60 \mathrm{~nm}$ & {$[33]$} \\
$\mathrm{Au}$ & $1025 \mathrm{~nm}$ & $450 \mathrm{fs}$ & $10-130 \mu \mathrm{J}$ & $7-50 \mathrm{~nm}$ & {$[27]$} \\
$\mathrm{Ag}$ & 355 and $532 \mathrm{~nm}$ & $6 \mathrm{~ns}$ and $18 \mathrm{ps}$ & 10 and $2-3 \mathrm{~mJ}$ & $5-60 \mathrm{~nm}$ & {$[28]$} \\
$\mathrm{Au}$ & $355 \mathrm{~nm}$ & $30 \mathrm{ps}$ & 6.3 and $17 \mathrm{~mJ} / \mathrm{cm}^{2}$ & NA & $\sim 10 \mathrm{~nm}$ or less \\
$\mathrm{Au} / \mathrm{Ag}$ & 532 and $1064 \mathrm{~nm}$ & $\sim 5 \mathrm{~ns}$ & $35 \mathrm{~mJ}$ & $\sim 26 \mathrm{~nm}$ & {$[20]$} \\
$\mathrm{Ag}$ & 355 and $1064 \mathrm{~nm}$ & 7 and $5 \mathrm{~ns}$ & $\mathrm{NA}$ & $\sim 10 \mathrm{~nm}$ & {$[34]$} \\
$\mathrm{Au}-\mathrm{Ag}$ alloy & 355 and $532 \mathrm{~nm}$ & $6 \mathrm{~ns}$ & 150 and $130 \mathrm{~mJ}$ & {$[35]$} & \\
\hline
\end{tabular}

NA: not applicable.

In this study, we produced Ag nanoparticles using laser pulse widths of femtoseconds to nanoseconds. Nanoparticle size and distribution were analyzed. With regard to the laser pulse width, we simulated the dispersion and energy absorption of the laser by the nanoparticles using wave optics.

\section{Background}

\subsection{Effect of Laser Pulse Width}

Irradiation of a solid target in a liquid with a laser beam results in the generation of metal flakes by laser fragmentation. The particles are refined by irradiation with the laser beam a second time. The laser beam reduces the size of the generated nanoparticles or produces particles with a specific size distribution. The interaction of the laser beam with the liquid also affects particle formation. Bubbles and cavitation result from laser-induced plasma generation and affect nanoparticle fragmentation [36,37]. In addition, the aqueous solution influences the formation of clusters of nanoparticles [38]. 
Nanoparticle size and dispersion can be controlled by varying the laser parameters. The wavelength of the laser pulse also affects nanoparticle generation [20]. When a laser of a different wavelength with the same pulse width is used, the fragmentation effect increases with decreasing pulse wavelengths. This is because the shorter the pulse wavelength, the greater the absorption cross section [28]. In addition, liquids other than deionized water may be used, and they can contribute to nanoparticle formation and stability $[16,20]$. Another study attempted to control the size of nickel (Ni) nanoparticles by using water containing other nanoparticles such as silica particles [39].

Laser pulse width is a predominant determinant of nanoparticle size and distribution. Laser pulse widths in the femtosecond, picosecond, and nanosecond regions can be used to induce laser fragmentation. Several studies have evaluated the interactions of picosecond or femtosecond laser pulses with materials $[27,32]$. Such ultrafast laser pulses cause nonlinear multiphoton absorption due to their high peak power. Because of this, energy cannot be transferred from the electrons to the ion grids in femtoseconds or picoseconds, which enables the processing of materials that are little affected by heat [40]. Irradiation with a nanosecond pulsed laser beam results in thermal diffusion at the particle surface. It is important to compare diffusion time and pulse width. In general, thermal diffusion in metal nanoparticles requires 50 to $100 \mathrm{ps,} \mathrm{and} \mathrm{the} \mathrm{diffusion} \mathrm{time} \mathrm{can} \mathrm{be} \mathrm{used} \mathrm{to} \mathrm{classify}$ laser fragmentation mechanisms into short and long pulse-width categories [41].

At a pulse width of less than a few picoseconds, the electron and phonon temperatures in the particle are different. In this case, to determine the particle temperature distribution, a model with different electron and phonon temperatures should be applied [42,43]. Also, because the laser beam absorption time is shorter than that of heat diffusion, it is appropriate to assume that the boundary condition of particles is adiabatic. In this model, the energy absorption by laser irradiation is a function of the fluence and absorption cross-sectional area of the laser [44]. This correlation affects the electron temperature and phonon temperature of the nanoparticles; that is, as the pulse width decreases, the peak laser power and the electron temperature increase. This results in a release of electrons from the particle; moreover, a Coulomb explosion may occur [28,33]. Coulomb explosions are a major mechanism of femtosecond laser fragmentation. When the pulse width increases from femtoseconds to picoseconds, the energy transfer tends to resolve the energy imbalance in the nanoparticles. In this case, the effect of Coulomb explosions in the particles decreases [45]. Near-field enhancement at the nanoparticle surface also occurs using ultrafast laser pulses [46]. When a nanostructure is irradiated with a short laser pulse, the electromagnetic field is amplified at the point at which an abrupt change in shape occurs. This results in a sudden energy concentration at that point, leading to particle fragmentation [47].

A model with constant electron and phonon temperatures can be used for lasers with nanosecond pulse widths. Also, because there is enough time for thermal diffusion within the particle, the heat at the interface interacts with the surroundings during laser irradiation. Therefore, the photothermal effect becomes dominant [20,48,49]. Melting, evaporation, or both occur at the particle surface as a result of the temperature imbalance between the particle surface and interior, which likely also causes particle fragmentation.

The three above-mentioned mechanisms of laser fragmentation are summarized in Figure 1. The effect on nanoparticle formation of mechanical shockwaves generated by laser-induced generation of plasma is shown in Figure 2. 


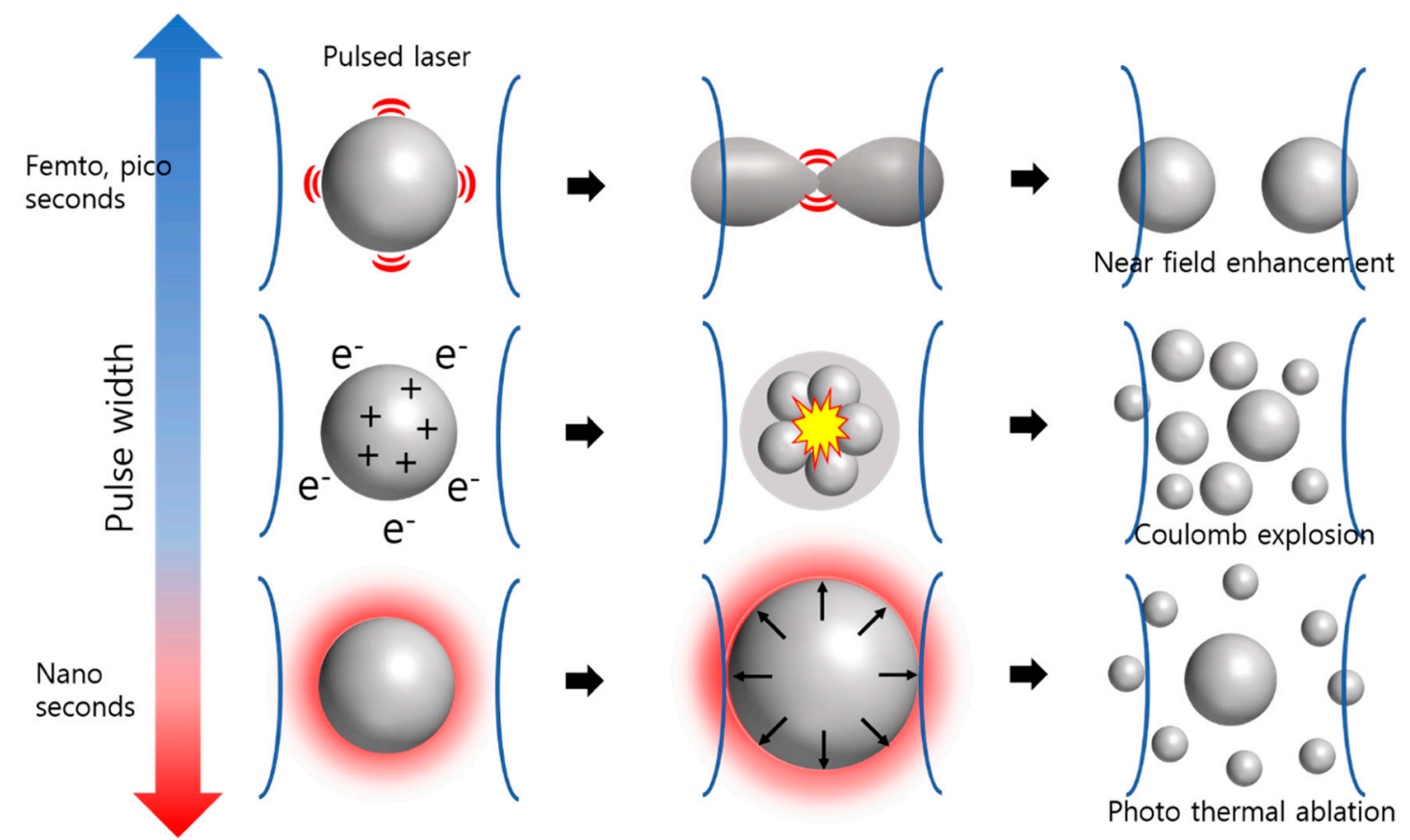

Figure 1. Mechanisms causing laser fragmentation for different laser pulse widths.
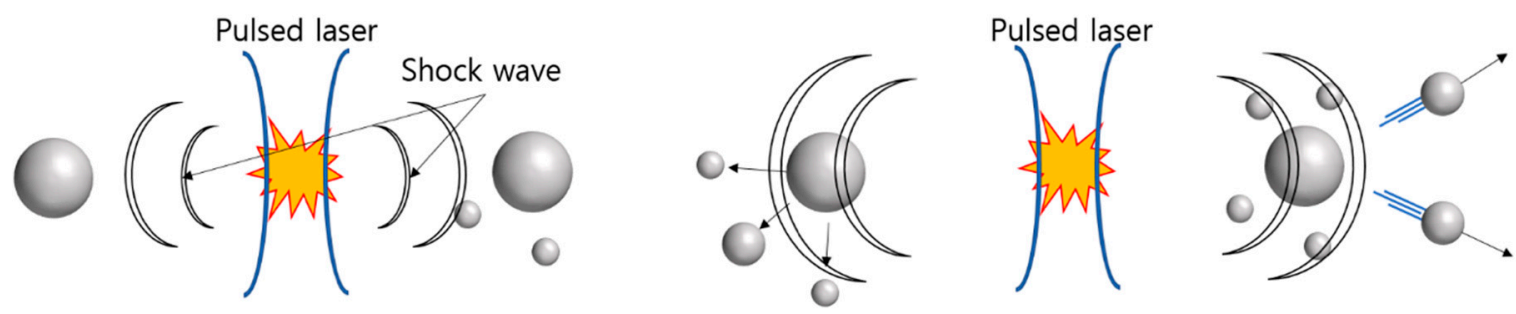

Figure 2. Effect of shockwave propagation on laser fragmentation.

\subsection{Simulation of Laser Fragmentation in the Nanosecond Region Using the Wave-Optic Theory}

The appropriate method for a numerical analysis of laser fragmentation differs depending on the pulse width. At femtosecond pulse widths, the mechanism of laser fragmentation can be indirectly explained by the temperature distribution of electrons and phonons [30,50]. At nanosecond pulse widths, photothermal ablation occurs. The interaction between the laser and the nanoparticles can be evaluated using wave optics.

Upon contacting a particle, the laser beam is scattered. The resulting energy waves are absorbed by the particles in a nonuniform manner because of their dispersion and interference effects. By numerically confirming the energy absorption by the particle, laser fragmentation due to nanosecond laser pulse widths can be indirectly analyzed. In wave optics, the energy absorption due to intra-particle dispersion of the laser beam is interpreted as an optical interaction. For this purpose, optical properties, such as the complex refractive index of nanoparticles whose diameter is less than the wavelength of the laser beam, should be evaluated. Unlike the surface of bulk Ag or Au, the absorption of laser energy by submicron Ag or Au particles is high, which can be explained by Mie theory $[20,51]$. 


\section{Numerical Simulation and Experimental Setup}

\subsection{Numerical Analysis}

To numerically analyze the interaction between a nanosecond pulse-width laser beam and a nanoparticle, a simulation was carried out using COMSOL ver. 5.2 software (COMSOL, Inc., Burlington, MA, USA). To validate the simulation, the results were compared with an analytical solution based on Mie theory in our prior research [52].

As described in the previous chapter, in the nanosecond region, the difference in the photothermal effect according to the pulse width can be numerically confirmed by the dispersion of the wave and its absorption by the particle. For the simulation, pulse widths of 4, 32, 64, and 100 ns were used. The simulation was performed using a water-encapsulated virtual Ag nanoparticle with a diameter of $100 \mathrm{~nm}$. Regarding the optical properties, the complex refractive index according to wavelength was evaluated using the optical property database in COMSOL 5.3. The complex refractive index of a particle can be used to confirm the behavior of a wave in that particle, and is related to energy absorption.

\subsection{Setup for Laser Fragmentation}

The laser sources were an ultrafast laser (PHAROS; Light Conversion, Vilnius, Lithuania) and a nanosecond laser (YLP-1; IPG Photonics, Oxford, MA, USA) with an adjustable pulse width. The ultrafast laser beam was generated through mode locking and amplification of chirped pulses. The pulse width of the laser beam was $164 \mathrm{fs}$ and $5 \mathrm{ps}$ at a wavelength of $1070 \mathrm{~nm}$. The nanosecond laser generated a laser pulse using a fiber resonance system, and the pulse width was 4, 32, 64, and $100 \mathrm{~ns}$ at a wavelength of $1070 \mathrm{~nm}$. The laser-related process parameters are shown in Table 2. To assess the effect of the laser pulse width, it is important that the energy doses be as similar as possible, within the limits of the experimental setup. In this experiment, the fluence of the femtosecond, picosecond, and nanosecond laser beams was set in the range from 0.10 to $0.13 \mathrm{~mJ} / \mathrm{cm}^{2}$.

Table 2. Laser parameters.

\begin{tabular}{cccc}
\hline Pulse Width & Femtosecond Regime & Picosecond Regime & Nanosecond Regime \\
\hline Fluence $\left(\mathrm{mJ} / \mathrm{cm}^{2}\right)$ & 0.13 & 0.13 & 0.10 \\
Repetition rate $(\mathrm{kHz})$ & 200 & 200 & 500 \\
Delay time $(\mu \mathrm{s})$ & 5 & 5 & 2 \\
Pulse duration & $164 \mathrm{fs}$ & $5 \mathrm{ps}, 32,64$, and $100 \mathrm{~ns}$ \\
Wavelength $(\mathrm{nm})$ & 1070 & 1070 & 1070 \\
Process time $(\mathrm{min})$ & 10 & 10 & 10 \\
\hline
\end{tabular}

The laser fragmentation setup is shown in Figure 3. The Ag target in distilled water was irradiated with a laser beam, which resulted in the production of nanoparticles. The emitted laser beam was controlled by a galvanometer scanner. The target was irradiated with the laser beam in a scanning pattern for $10 \mathrm{~min}$. The laser beam had a Gaussian energy distribution and a spot size of 20 to $40 \mu \mathrm{m}$. Ag nanoparticles were synthesized primarily during laser ablation, as well as by secondary laser fragmentation in the solution. Ag nanoparticles generated by laser fragmentation form clusters over time. The colloids thus generated can be transformed to stable nanoparticles by further laser irradiation; however, this was not performed in this study. Ag nanoparticles were analyzed at least one day after laser fragmentation. The nanoparticles were dried and visualized by transmission electron microscopy (TEM; JEM-2010; JEOL, Tokyo, Japan) and scanning electron microscopy (SU8010; Hitachi, Tokyo, Japan). In addition, the Ag nanoparticle size distribution was determined using a particle size analyzer (ELS-Z PLUS; Otsuka Electronics, Osaka, Japan); 300 to 500 nanoparticle samples were used per laser parameter. 


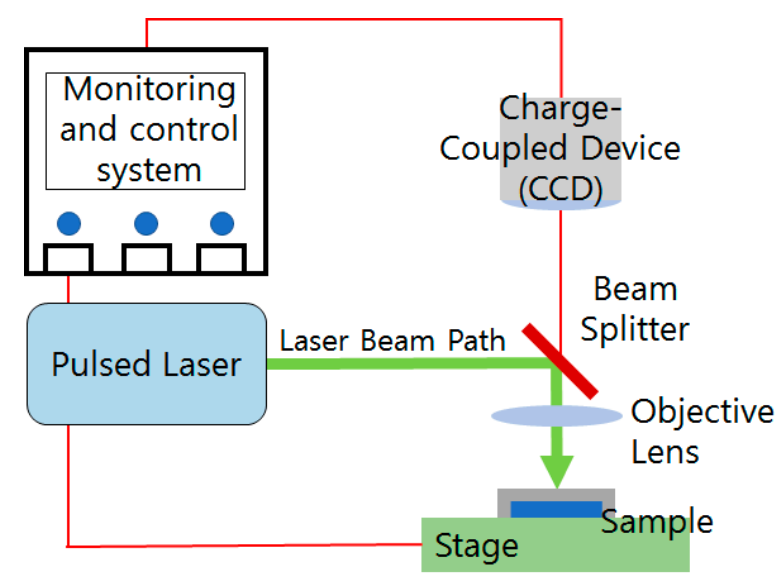

Figure 3. Schematic diagram of the experimental setup.

\section{Results and Discussion}

\subsection{Simulation of Nanoparticle Temperature Distribution According to the Laser Pulse Width}

The distributions of the energy absorption rates were simulated according to the laser pulse width. Most of the irradiated energy was absorbed by the several-nanometer-thick surface layer of the nanoparticles. This may result in peeling due to thermal strain between the surface and the inner layers. The thermal effect of nanosecond laser pulses is thought to result in fragmentation because of the weaker force of the outermost atoms in the particles [31]. However, our simulation showed that the thermal effect of energy dispersion and interference on the outer surface is also important. The inhomogeneous distribution of energy absorption in nanoparticles leads to a partial deformation or evaporation of the surface, which can accelerate the fragmentation. In the nanosecond region, the thermal effect on nanoparticles increases with decreasing pulse widths. Assuming the same energy per pulse, when the nanoparticle surface is irradiated with a laser of pulse widths 4, 32, 64, and $100 \mathrm{ns,}$ the surface temperature increases, which affects particle fragmentation. According to the simulation, the shorter the laser width pulse, the greater is the inhomogeneous increase in the energy absorption rate. The total energy absorption according to previous results was numerically calculated (Figure 4). The energy absorption by the nanoparticles increased markedly with decreasing pulse widths in the nanosecond range. Therefore, the rate of nanoparticle decomposition increased with decreasing laser pulse widths, leading to the production of smaller nanoparticles.

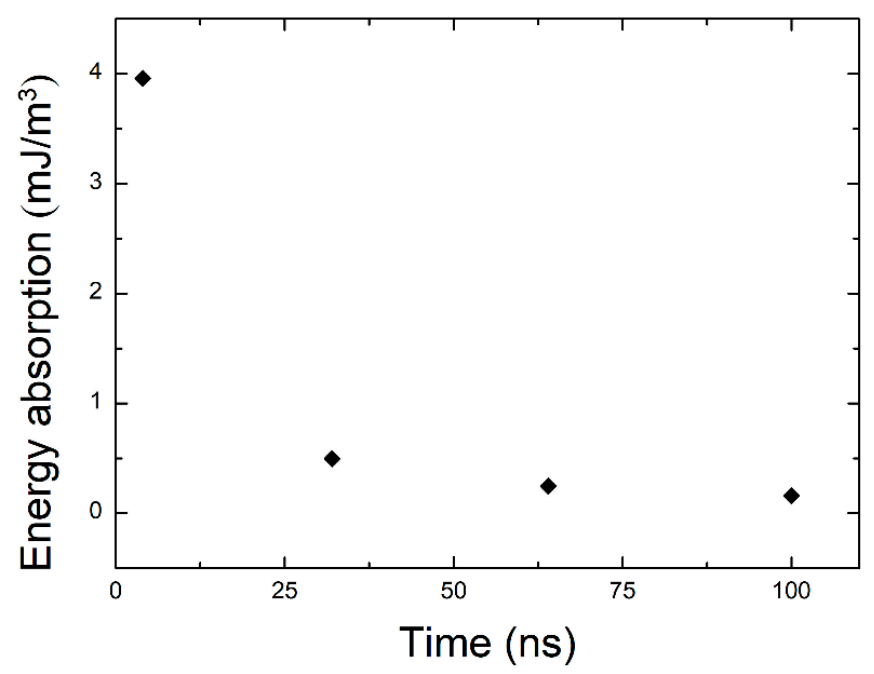

Figure 4. Total energy absorption of nanoparticles as a function of the pulse width. 


\subsection{Nanoparticle Size Distribution}

Nanoparticle shape was visualized after laser fragmentation for $\geq 1 \mathrm{~h}$. Solutions of nanoparticles produced using femtosecond and picosecond laser pulse widths were yellow in color, and no precipitate was present. However, solutions of nanoparticles produced using nanosecond laser pulse widths were gray in color, and clusters had precipitated. The Ag nanoparticles thus generated can change physically or chemically as time passes. Ag nanoparticles can generate colloids and can be converted into oxides such as $\mathrm{AgO}$ or $\mathrm{Ag}_{2} \mathrm{O}$ as they bind to oxygen. These issues are observed not only in nanoparticles generated by laser fragmentation, but also in other nanoparticle production processes.

Spherical and cylindrical Ag nanoparticles, some in the form of linked clusters, were detected by TEM (Figure 5). In addition, the nanoparticle size distribution was evaluated (Figure 6). Nanoparticles produced using femtosecond laser pulse widths were small, having a diameter of around $25 \mathrm{~nm}$ (Figures 5a and 6a). In the nanosecond region, as the pulse width increased, the nanoparticle size distribution became broader. Therefore, the size distribution of nanoparticles is determined by the laser pulse width.

To analyze nanoparticle generation using femtosecond laser pulse widths, the mechanisms of nanoparticle generation according to pulse width must be evaluated. These mechanisms are near-field ablation, Coulomb explosion, photothermal ablation, and mechanical breakdown due to shockwaves. Among these, near-field enhancement and Coulomb explosion are likely involved in nanoparticle generation by femtosecond laser pulse widths. In addition, the laser beam-induced shockwave affects the generation of nanoparticles and the formation of clusters. Using femtosecond laser pulse widths, spherical nanoparticles were created by Coulomb explosion, and nonspherical nanoparticles by near-field enhancement. However, distinguishing between these two mechanisms is problematic because of the nonuniform nanoparticle shape, which is a result of both the interaction of the laser beam with the target and laser fragmentation.

The shapes of nanoparticles generated using picosecond and $164 \mathrm{fs}$ laser pulses were similar (Figure $5 \mathrm{~b}$ ). In addition, their size distribution was similar to that of nanoparticles produced using femtosecond pulse widths (Figure 6b). Thermal diffusion within the particle resulting from electron-phonon scattering occurs at laser pulse widths of around a few picoseconds [41]. However, thermal diffusion at the boundary takes several hundred picoseconds [28,41]. Therefore, heat transfer at the particle surface does not occur during laser irradiation for $5 \mathrm{ps}$. Therefore, the mechanism of laser fragmentation with a pulse width of $5 \mathrm{ps}$ is similar to that of lasers with femtosecond pulse widths.

The energy accumulation by particles subjected to successive laser beam pulses must also be considered. Based on its repetition rate, the laser pulse has a delay time of 2 to $5 \mu \mathrm{s}$. Thus, on the basis of the difference between delay time and laser pulse width, the particle energy accumulation is not significant and can be disregarded.

The irradiation with a nanosecond pulse-width laser beam produced particles of diameters from $25 \mathrm{~nm}$ to more than $200 \mathrm{~nm}$, which were in a linked-cluster form (Figure $5 \mathrm{c}-\mathrm{f}$ ). For lasers with nanosecond pulse widths, the shorter the pulse width, the smaller the maximum particle diameter. The broad particle size distribution is due to photothermal ablation. Our simulation showed that the thermal effect predominated at nanosecond laser pulse widths and induced peeling off of the outermost layers. By using this exfoliation process, it is possible to produce nanoparticles of various sizes. Moreover, laser-induced plasma generation and particle fragmentation due to the shockwave are also involved in particle formation.

The mean diameters of the nanoparticles produced using laser pulse widths of 4, 32, 64, and $100 \mathrm{~ns}$ were 75 85, 100 110, 95 105, and 120 130 nm, respectively as shown in Figure 7. The smallest value were seen for the femtosecond pulse, and the values tended to increase with the pulse width up to nanoseconds. The rate of diameter size change increased markedly when the pulse width changed from picosecond to nanosecond. The analysis of these results revealed that the region of particle size change according to the pulse width could be divided into the femtosecond and picosecond region, and the nanosecond region. This supports the fact that the mechanism of near-field enhancement and 
Coulomb explosion as described above and the mechanism of photothermal ablation are different in nanoparticle generation. In addition, the rate of increase of the particle size was larger in the nanosecond region, which is a characteristic of the laser thermal ablation related to the surface area. Conversely, it could be inferred that the surface area and fragmentation of the particles had a weak relationship in the femtosecond and picosecond regions.
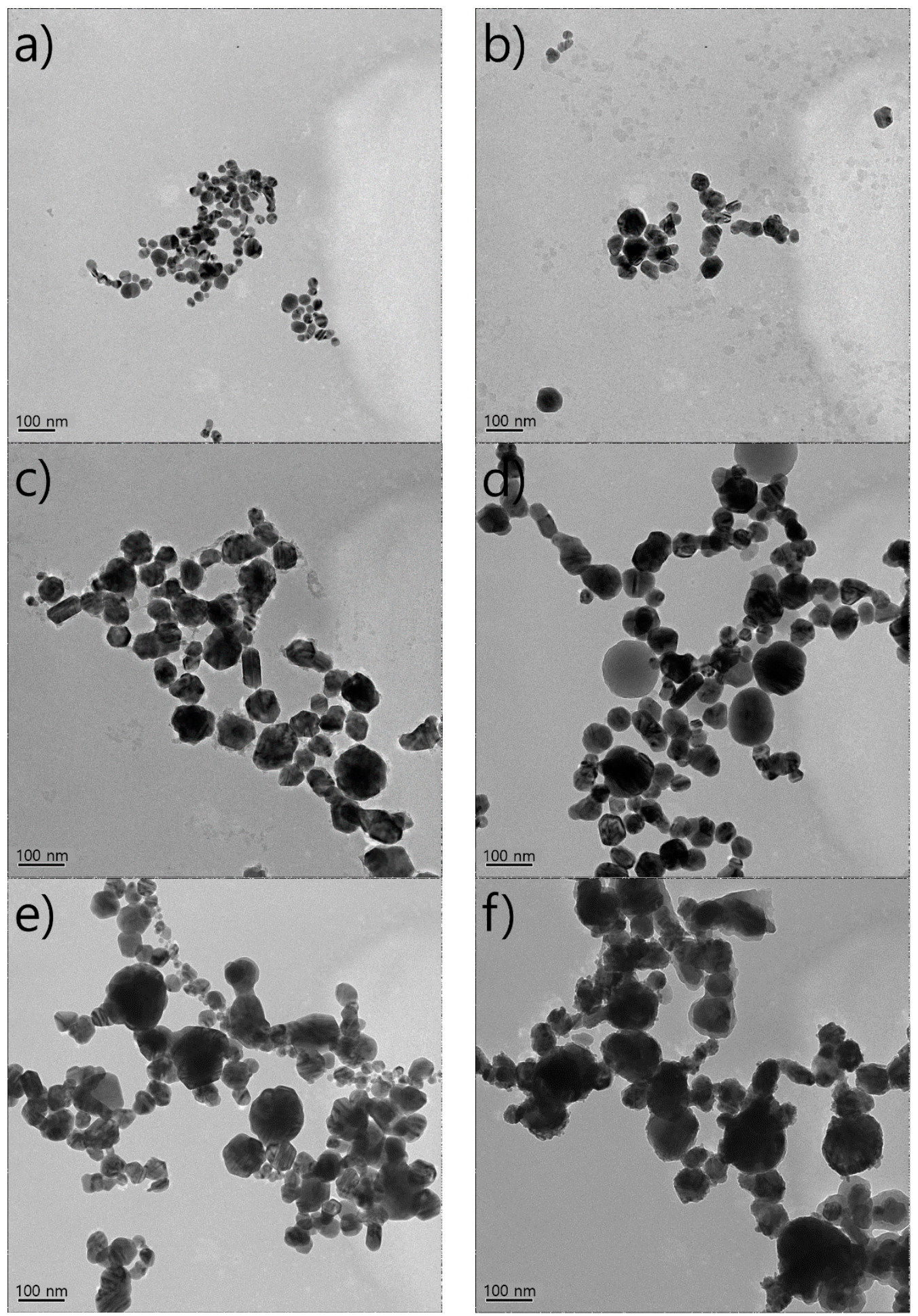

Figure 5. Transmission electron microscopy (TEM) images of Ag nanoparticles after being dried on a glass surface. (a) 164 fs, (b) 5 ps, (c) 4 ns, (d) 32 ns, (e) 64 ns, and (f) 100 ns pulse widths. 
The yield rate of nanoparticles according to the laser pulse width can be confirmed by two methods. The first is the yield rate according to the total mass of nanoparticles per unit area and unit time. The second is the yield rate according to the total number of nanoparticles per unit area and unit time. The nanoparticle weight production rate was higher using lasers with nanosecond pulse widths $\left(0.71 \times 10^{-15} \sim 2.79 \times 10^{-15} \mathrm{~g} / \mathrm{min}\right)$ compared to that obtained using lasers with femtosecond and picosecond pulse widths $\left(4.78 \times 10^{-17} \sim 8.06 \times 10^{-17} \mathrm{~g} / \mathrm{min}\right)$. However, this result is directly related to laser ablation of the target in water. As previously described, nanoparticles generated from nanosecond laser pulses have larger mean diameters. Therefore, even if the same number of nanoparticles is generated, the total mass of the nanoparticles generated from the nanosecond laser pulses is larger than that of the nanoparticles generated from the femtosecond or picosecond laser pulses. In our experiment, the number of nanoparticles generated for each parameter was measured to determine the particle generation rate according to the laser pulse width (Figure 8). This showed that the number of nanoparticles increased as the pulse width was shortened. Therefore, laser fragmentation is more efficient in the femtosecond or picosecond region. Considering these results, the laser pulse width that can be applied depends on the size of the nanoparticles required. Moreover, the change in nanoparticle size in relation to the pulse width is more sensitive in the nanosecond region.

a)

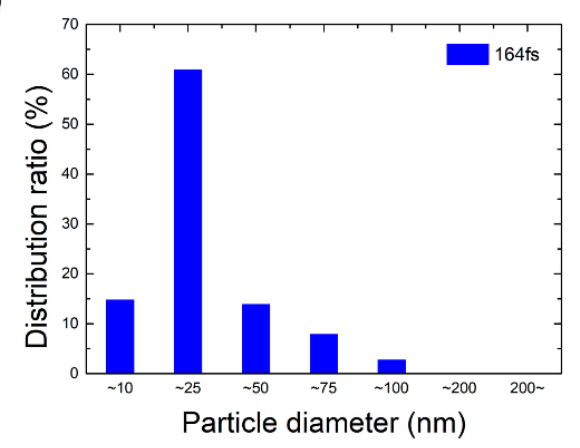

c)

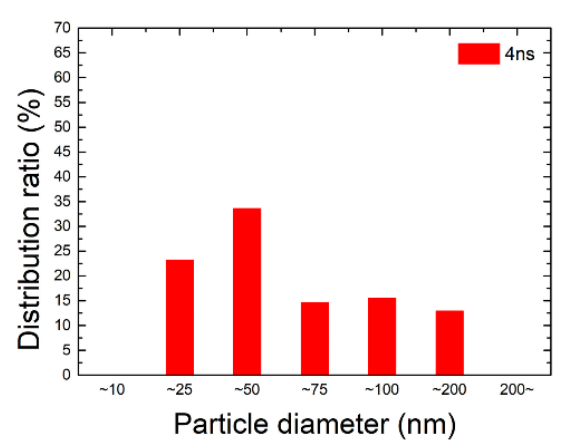

e)

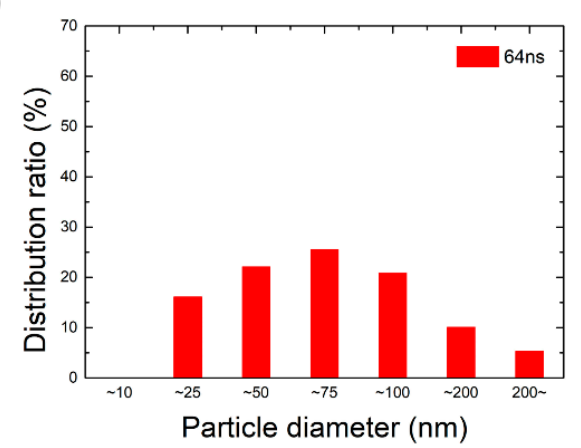

b)

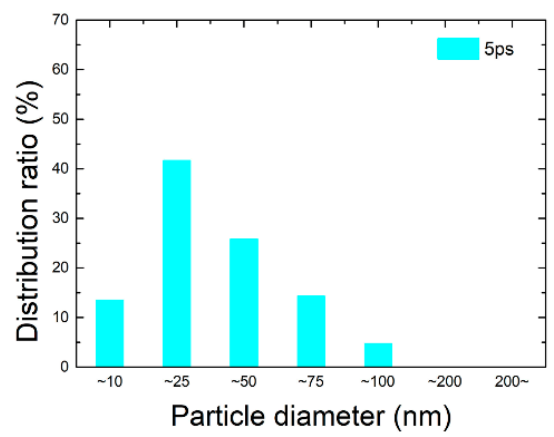

d)

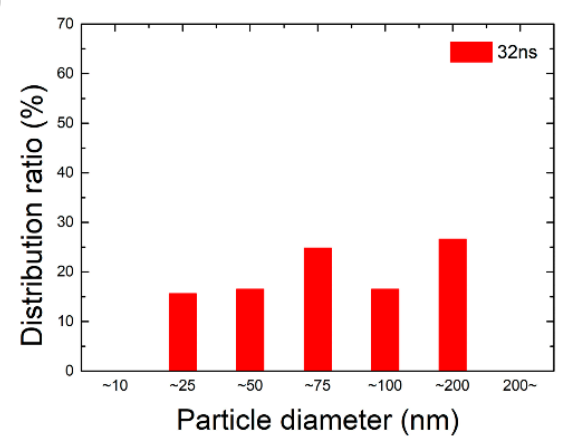

f)

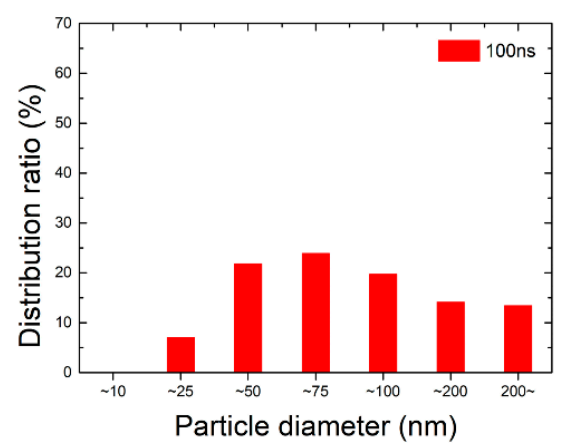

Figure 6. Size distribution of Ag nanoparticles. (a) 164 fs, (b) 5 ps, (c) 4 ns, (d) 32 ns, (e) 64 ns, and (f) 100 ns pulse widths. 


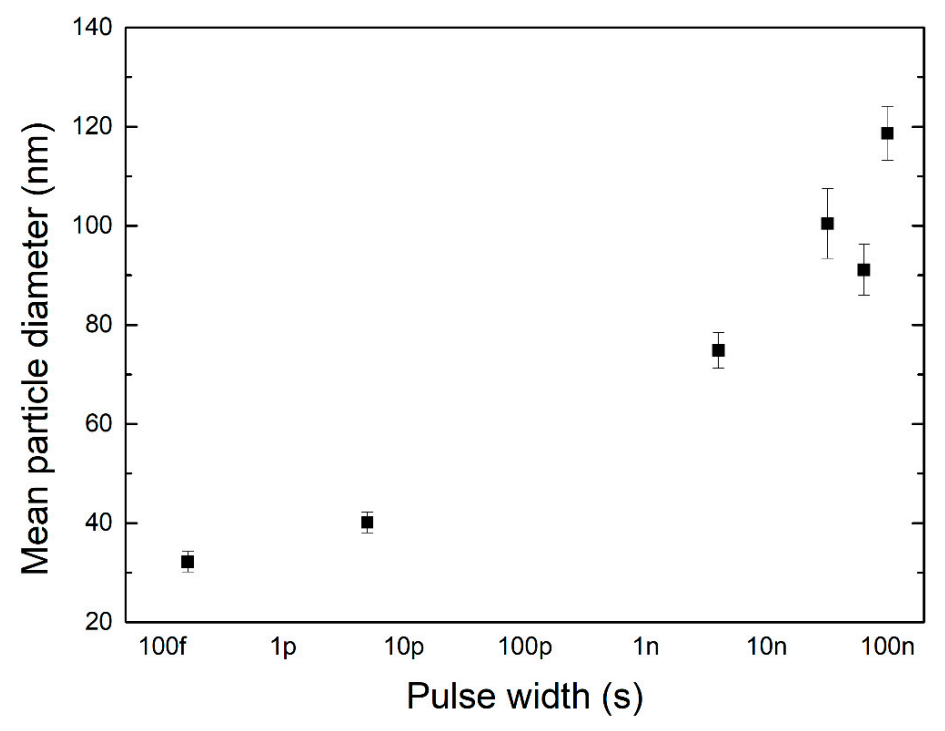

Figure 7. Mean nanoparticle diameter as a function of the pulse width.

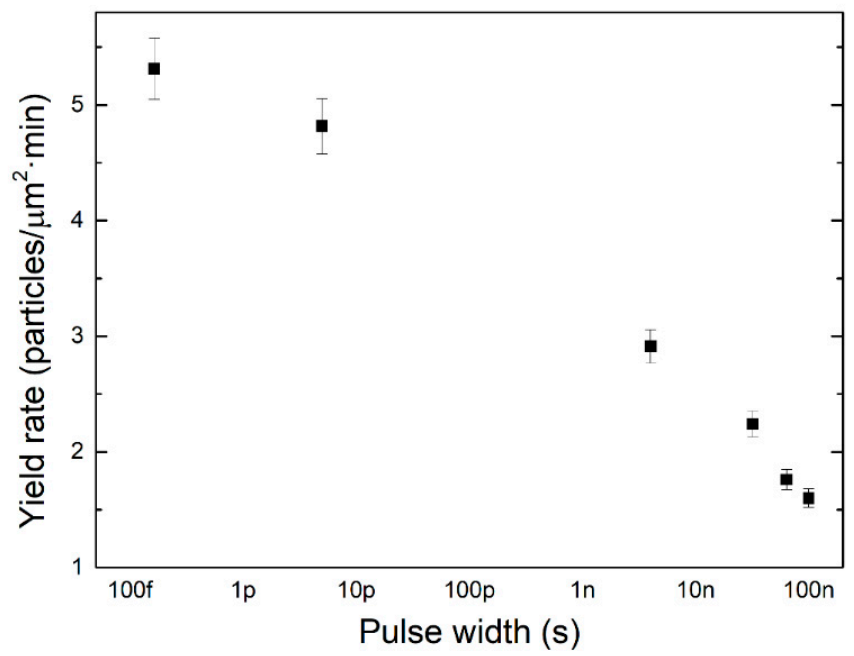

Figure 8. Nanoparticle yield rate as a function of the pulse width.

\section{Conclusions}

This study evaluated the production of Ag nanoparticles according to the laser pulse width. As the laser pulse width decreased, the average nanoparticle size decreased. The nanoparticles produced using femtosecond and picosecond laser pulse widths were found to include more $25 \mu \mathrm{m}$ particles than particles of other sizes, whereas, by using nanosecond laser pulse widths, nanoparticles ranging in size from 25 to $200 \mathrm{~nm}$ were produced. The rate of increase in the particle size is larger in the nanosecond region, which is seen as a characteristic of the laser thermal ablation related to the surface area. In comparison, in the femtosecond and picosecond regions, a larger number of nanoparticles was produced. This phenomenon can be interpreted on the basis that the shorter the pulse width, the more efficient the occurrence of fragmentation.

Acknowledgments: This study was supported by the Research Program funded by the SeoulTech(Seoul National University of Science and Technology).

Author Contributions: Hae Woon Choi and Joohan Kim conceived and designed the experiments; Jin-Woo Jeon and Sangwoo Yoon performed the experiments; Dave Farson and Sung-Hak Cho analyzed the data; Jin-Woo Jeon and Joohan Kim wrote the paper. 
Conflicts of Interest: The authors declare no conflict of interest.

\section{References}

1. Yguerabide, J.; Ygerabide, E.E. Light-scattering submicroscopic particles as highly fluorescent analogs and their use as tracer labels in clinical and biological applications. Anal. Biochem. 1998, 262, 137-156. [CrossRef] [PubMed]

2. Guo, X.; Guo, B.; Wang, Y.; Guan, S. Preparation of spherical metal-organic frameworks encapsulating Ag nanoparticles and study on its antibacterial activity. Mater. Sci. Eng. C 2017, 80, 698-707. [CrossRef]

3. Chatterjee, S.; Shariff, S.M.; Majumdar, J.D.; Choudhury, A.R. Development of nano-structured $\mathrm{Al}_{2} \mathrm{O}_{3}-\mathrm{TiB}_{2}-\mathrm{TiN}$ coatings by combined SHS and laser surface alloying. Int. J. Adv. Manuf. Technol. 2008, 38, 938-943. [CrossRef]

4. Raj, D.R.; Sudarsanakumar, C. Surface plasmon resonance based fiber optic sensor for the detection of cysteine using diosmin capped silver nanoparticles. Sens. Actuators A Phys. 2017, 253, 41-48. [CrossRef]

5. Middha, M.; Kumar, R.; Raina, K.K. Effects of chirality on optical and electro-optic behavior of nematic liquid crystals doped with functionalized silver nanoparticles. J. Mol. Liq. 2016, 219, 631-636. [CrossRef]

6. Hashimoto, Y.; Takeuchi, S.; Mitsunobu, S.; Ok, Y.S. Chemical speciation of silver (Ag) in soils under aerobic and anaerobic conditions: Ag nanoparticles vs. ionic Ag. J. Hazard. Mater. 2017, 322, 318-324. [CrossRef] [PubMed]

7. Gao, S.; Ueno, K.; Misawa, H. Plasmonic antenna effects on photochemical reactions. Acc. Chem. Res. 2011, 44, 251-260. [CrossRef] [PubMed]

8. Gratzel, M. Dye-sensitized solar cells. J. Photochem. Photobiol. C 2003, 4, 145-153. [CrossRef]

9. Mayer, A.C.; Scully, S.R.; Hardin, B.E.; Rowell, M.W.; McGehee, M.D. Polymer-based solar cells. Mater. Today 2007, 10, 28-33. [CrossRef]

10. Kamat, P.V. Quantum dot solar cells. Semiconductor nanocrystals as light harvesters. J. Phys. Chem. C 2008, 112, 18737-18753. [CrossRef]

11. Nikolay, T.; Larina, L.; Shevaleevskiy, O.; Ahn, B.T. Electronic structure study of lightly $\mathrm{Nb}_{-}$doped $\mathrm{TiO}_{2}$ electrode for dye-sensitized solar cells. Energy Environ. Sci. 2011, 4, 1480-1486. [CrossRef]

12. Jiang, C.; Leung, C.W.; Pong, P.W.T. Self-assembled thin films of $\mathrm{Fe}_{3} \mathrm{O}_{4}$-Ag composite nanoparticles for spintronic applications. Appl. Surf. Sci. 2017, 419, 692-696. [CrossRef]

13. He, W.; Ye, C. Flexible Transparent Conductive Films on the Basis of Ag Nanowires: Design and Applications: A Review. J. Mater. Sci. Technol. 2015, 31, 581-588. [CrossRef]

14. Oh, H.; Lee, M. Laser-direct fabrication of invisible Ag nanowire electrode pattern on flexible plastic substrate. Thin Solid Films 2017, 636, 375-383. [CrossRef]

15. Koleva, M.E.; Nedyalkov, N.N.; Fukata, N.; Jevasuwan, W.; Karashanova, D. Laser-assisted approach for synthesis of plasmonic Ag/ZnO nanostructures. Superlattices Microstruct. 2017, 109, 886-896. [CrossRef]

16. Moura, C.G.; Pereira, R.S.F.; Andritschky, M.; Lopes, A.L.B.; Silva, F.S. Effects of laser fluence and liquid media on preparation of small Ag nanoparticles by laser ablation in liquid Original research article. Opt. Laser Technol. 2017, 97, 20-28. [CrossRef]

17. Vella, P.C.; Dimov, S.S.; Brousseau, E.; Whiteside, B.R. A new process chain for producing bulk metallic glass replication masters with micro-and nano-scale features. Int. J. Adv. Manuf. Technol. 2015, 76, 523-543. [CrossRef]

18. Kuo, C.G.; Chao, C.G. A novel method of centrifugal processing for the synthesis of lead-bismuth eutectic alloy nanospheres and nanowires. Int. J. Adv. Manuf. Technol. 2007, 32, 468-472. [CrossRef]

19. Chang, H.; Jwo, C.S.; Fan, P.S.; Pai, S.H. Process optimization and material properties for nanofluid manufacturing. Int. J. Adv. Manuf. Technol. 2007, 34, 300-306. [CrossRef]

20. Kawasaki, M.; Nishimura, N. 1064-nm laser fragmentation of thin Au and Ag flakes in acetone for highly productive pathway to stable metal nanoparticles. Appl. Surf. Sci. 2006, 253, 2208-2216. [CrossRef]

21. Serkov, A.A.; Kuzmin, P.G.; Shafeev, G.A. Laser-induced agglomeration of gold and silver nanoparticles dispersed in liquid. Chem. Phys. Lett. 2016, 647, 68-72. [CrossRef]

22. Amendola, V.; Scaramuzza, S.; Carraro, F.; Cattaruzza, E. Formation of alloy nanoparticles by laser ablation of $\mathrm{Au} / \mathrm{Fe}$ multilayer films in liquid environment. J. Colloid Interface Sci. 2017, 489, 18-27. [CrossRef] [PubMed] 
23. Swiatkowska-Warkocka, Z.; Pyatenko, A.; Koshizaki, N.; Kawaguchi, K. Synthesis of various 3D porous gold-based alloy nanostructures with branched shapes. J. Colloid Interface Sci. 2016, 483, 281-286. [CrossRef] [PubMed]

24. Tomko, J.; O’Malley, S.M.; Trout, C.; Naddeo, J.J.; Bubb, D.M. Cavitation bubble dynamics and nanoparticle size distributions in laser ablation in liquids. Colloid Surf. A 2017, 522, 368-372. [CrossRef]

25. Tanabe, R.; Nguyen, T.T.P.; Sugiura, T.; Ito, Y. Bubble dynamics in metal nanoparticle formation by laser ablation in liquid studied through high-speed laser stroboscopic videography. Appl. Surf. Sci. 2015, 351, 327-331. [CrossRef]

26. Takeda, Y.; Mafuné, F. Formation of wide bandgap cerium oxide nanoparticles by laser ablation in aqueous solution. Chem. Phys. Lett. 2014, 599, 110-115. [CrossRef]

27. Maximova, K.; Aristov, A.; Sentis, M.; Kabashin, A.V. Size-controllable synthesis of bare gold nanoparticles by femtosecond laser fragmentation in water. Nanotechnology 2015, 26, 065601. [CrossRef] [PubMed]

28. Kamat, P.V.; Flumiani, M.; Hartland, G.V. Picosecond Dynamics of Silver Nanoclusters. Photoejection of Electrons and Fragmentation. J. Phys. Chem. B 1998, 102, 3123-3128. [CrossRef]

29. Kohsakowski, S.; Santagata, A.; Dell'Aglio, M.; de Giacomo, A.; Barcikowski, S.; Wagener, P.; Gökce, B. High productive and continuous nanoparticle fabrication by laser ablation of a wire-target in a liquid jet. Appl. Surf. Sci. 2017, 403, 487-499. [CrossRef]

30. Leitz, K.H.; Redlingshöfer, B.; Reg, Y.; Otto, A.; Schmidt, M. Metal Ablation with Short and Ultrashort Laser Pulses. Phys. Procedia 2011, 12, 230-238. [CrossRef]

31. Link, S.; Burda, C.; Mohamed, M.B.; Nikoobakht, B.; El-Sayed, M.A. Laser Photothermal Melting and Fragmentation of Gold Nanorods: Energy and Laser Pulse-Width Dependence. J. Phys. Chem. A 1999, 103, 1165-1170. [CrossRef]

32. Inasawa, S.; Sugiyama, M.; Noda, S.; Yamaguchi, Y. Spectroscopic Study of Laser-Induced Phase Transition of Gold Nanoparticles on Nanosecond Time Scales and Longer. J. Phys. Chem. B 2006, 110, 3114-3119. [CrossRef] [PubMed]

33. Werner, D.; Furube, A.; Okamoto, T.; Hashimoto, S. Femtosecond Laser-Induced Size Reduction of Aqueous Gold Nanoparticles: In Situ and Pump-Probe Spectroscopy Investigations Revealing Coulomb Explosion. J. Phys. Chem. C 2011, 115, 8503-8512. [CrossRef]

34. Bae, C.H.; Nam, S.H.; Park, S.M. Formation of silver nanoparticles by laser ablation of a silver target in $\mathrm{NaCl}$ solution. Appl. Surf. Sci. 2002, 197, 628-634. [CrossRef]

35. Peng, Z.; Spliethoff, B.; Tesche, B.; Walther, T.; Kleinermanns, K. Laser-Assisted Synthesis of Au-Ag Alloy Nanoparticles in Solution. J. Phys. Chem. B 2006, 110, 2549-2554. [CrossRef] [PubMed]

36. Vogel, A.; Noack, J.; Nahen, K.; Theisen, D.; Busch, S.; Parlitz, U.; Hammer, D.X.; Noojin, G.D.; Rockwell, B.A.; Birngruber, R. Energy balance of optical breakdown in water at nanosecond to femtosecond time scales. Appl. Phys. B 1999, 68, 271-280. [CrossRef]

37. Reich, S.; Schönfeld, P.; Wagener, P.; Letzel, A.; Ibrahimkutty, S.; Gökce, B.; Barcikowski, S.; Menzel, A.; Rolo, T.D.S.; Plech, A. Pulsed laser ablation in liquids: Impact of the bubble dynamics on particle formation. J. Colloid Interface Sci. 2017, 489, 106-113. [CrossRef] [PubMed]

38. Inasawa, S.; Sugiyama, M.; Yamaguchi, Y. Laser-Induced Shape Transformation of Gold Nanoparticles below the Melting Point: The Effect of Surface Melting. J. Phys. Chem. B 2005, 109, 3104-3111. [CrossRef] [PubMed]

39. Mafuné, F.; Okamoto, T.; Ito, M. Surfactant-free small Ni nanoparticles trapped on silica nanoparticles prepared by pulsed laser ablation in liquid. Chem. Phys. Lett. 2014, 591, 193-196. [CrossRef]

40. Neuenschwander, B.; Jaeggi, B.; Schmid, M. From fs to sub-ns: Dependence of the material removal rate on the pulse duration for metals. Phys. Procedia 2013, 41, 794-801. [CrossRef]

41. Ahmadi, T.S.; Logunov, S.L.; El-Sayed, M.A. Picosecond Dynamics of Colloidal Gold Nanoparticles. J. Phys. Chem. 1996, 100, 8053-8056. [CrossRef]

42. Eesley, G.L. Observation of nonequilibrium electron heating in copper. Phys. Rev. Lett. 1983, 517, $2140-2143$. [CrossRef]

43. Link, S.; Burda, C.; Mohamed, M.B.; Nikoobakht, B.; El-Sayed, M.A. Femtosecond transient-absorption dynamics of colloidal gold nanorods: Shape independence of the electron-phonon relaxation time. Phys. Rev. B 2000, 61, 6086. [CrossRef]

44. Besner, S.; Kabashin, A.V.; Meunier, M. Fragmentation of colloidal nanoparticles by femtosecond laser-induced supercontinuum generation. Appl. Phys. Lett. 2006, 89, 233122. [CrossRef] 
45. Werner, D.; Hashimoto, S. Improved Working Model for Interpreting the Excitation Wavelength- and Fluence-Dependent Response in Pulsed Laser-Induced Size Reduction of Aqueous Gold Nanoparticles. J. Phys. Chem. C 2011, 115, 5063-5072. [CrossRef]

46. Plech, A.; Kotaidis, V.; Lorenc, M.; Boneberg, J. Femtosecond laser near-field ablation from gold nanoparticles. Nat. Phys. 2006, 2, 44-47. [CrossRef]

47. Ueno, K.; Juodkazis, S.; Shibuya, T.; Yokota, Y.; Mizeikis, V.; Sasaki, K.; Misawa, H. Nanoparticle Plasmon-Assisted Two-Photon Polymerization Induced by Incoherent Excitation Source. J. Am. Chem. Soc. 2008, 130, 6928-6929. [CrossRef] [PubMed]

48. Werner, D.; Hashimoto, S.; Uwada, T. Remarkable Photothermal Effect of Interband Excitation on Nanosecond Laser-Induced Reshaping and Size Reduction of Pseudospherical Gold Nanoparticles in Aqueous Solution. Langmuir 2010, 26, 9956-9963. [CrossRef] [PubMed]

49. Zedan, A.F.; Moussa, S.; Terner, J.; Atkinson, G.; El-Shall, M.S. Ultrasmall gold nanoparticles anchored to graphene and enhanced photothermal effects by laser irradiation of gold nanostructures in graphene oxide solutions. ACS Nano 2013, 7, 627-636. [CrossRef] [PubMed]

50. Neuenschwander, B.; Jaeggi, B.; Schmid, M.; Hennig, G. Surface structuring with ultra-short laser pulses: Basics, limitations and needs for high throughput. Phys. Procedia 2014, 56, 1047-1058. [CrossRef]

51. Kawasaki, M.; Masuda, K. Laser Fragmentation of Water-Suspended Gold Flakes via Spherical Submicroparticles to Fine Nanoparticles. J. Phys. Chem. B 2005, 109, 9379-9388. [CrossRef] [PubMed]

52. Choi, H.W. Laser Beam Scattering Analysis inside Porous Materials by FEM. In Future Information Technology, Application, and Service; Park, J., Leung, V., Wang, C.L., Shon, T., Eds.; Lecture Notes in Electrical Engineering; Springer: Dordrecht, The Netherlands, 2012; Volume 164, pp. 603-614. ISBN 978-94-007-4515-5.

(C) 2018 by the authors. Licensee MDPI, Basel, Switzerland. This article is an open access article distributed under the terms and conditions of the Creative Commons Attribution (CC BY) license (http:/ / creativecommons.org/licenses/by/4.0/). 